03

\title{
Эпитропно-жидкокристаллический слой гексадекана в реологической модели гетерофазной прослойки
}

\author{
(C) Б.А. Алтоиз, А.Ф. Бутенко, С.В. Кириян \\ Одесский национальный университет им. И.И. Мечникова, \\ 65082 Одесса, Украина \\ e-mail: altoiz41@gmail.com
}

(Поступило в Редакцию 20 июля 2016 г. В окончательной редакции 20 июня 2017 г.)

Гидродинамическая модель гетерофазной прослойки жидкости, включающая эпитропный жидкокристаллический (ЭЖК) слой, дополнена его структурно-реологической моделью. Применение моделей и разработанной методики обработки результатов измерений вязкости прослоек $н$-гексадекана в триаде трения позволило установить детально свойства его ЭЖК слоя и слоя „перекрытого“: равновесную толщину и вязкость, их изменение с температурой, энергии активации вязкого течения.

DOI: 10.21883/JTF.2018.01.45473.2005

\section{Введение}

Изучение свойств полимолекулярного ориентационно упорядоченного приповерхностного эпитропно ${ }^{1-}$ жидкокристаллического (ЭЖК [1-3]) слоя необходимо для развития теоретических представлений о природе этого состояния [3-6], усовершенствования его количественной физической модели [7] и, в частности ЭЖК слоев $\boldsymbol{н}$-алканов как основ минеральных смазочных масел [8-10], для решения практической задачи управления процессами трения в механизмах [11,12].

Ввиду малой $(\sim 4 \mu \mathrm{m}$ и менее $)$ толщины $d_{s}$ ЭЖК слоев прямое их изучение затруднительно, поэтому в оптических $[2,3,5,6,12,13]$ и реологических [12,14-16] экспериментах обычно исследовались гетерофазные (содержащие ЭЖК слои и пространственно отделенную от них „объемную“ жидкость) прослойки толщиной $D \sim 1.5-10 \mu \mathrm{m}$, а для установления характеристик слоев использовалась определенная структурная модель прослойки, в которой заложены параметры слоя $d_{s}$ и вязкость $\mu$. При расчете последних в реологических опытах измерялась „эффективная“ вязкость $\eta_{\mathrm{eff}}$ прослойки и применялись дополнительно различные гидродинамические модели слоя с постоянной вязкостью, зависящей от расстояния до подложки, от интенсивности течения и др., но собственно структура ЭЖК слоя при этом не рассматривалась. Предлагаемые [3-6] механизмы организации ЭЖК фазы в должной степени не описывали экспериментально установленные ее свойства.

\section{Механизм образования и структурно-реологическая модель ЭЖК слоя}

Согласно последним представлениям [7] об ЭЖК фазе, ее существование обусловлено сцеплением с актив-

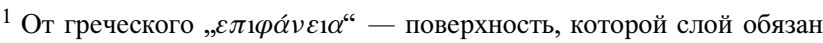
своим происхождением.
}

ными центрами лиофильной подложки приповерхностных флуктуационных ассоциатов [17] жидкости. „Ворс“ таких нитевидных (средней длины $d_{s}$ ) молекулярных ассоциатов вместе с неассоциированной жидкостью между ними и представляет собой ЭЖК слой. ${ }^{2}$ Он существует вблизи температуры кристаллизации жидкости $T_{\text {cr }}$ в небольшом интервале $\Delta T=\left(T-T_{\mathrm{cr}}\right) \sim 30-40 \mathrm{~K}$. С ростом $\Delta T$ происходит процесс десорбции ассоциатов, толщина „ворса“ и доля последних в ЭЖК слое уменьшаются, а доля изотропной жидкости в нем возрастает. ${ }^{3}$

Это позволяет структурно-реологическую модель собственно ЭЖК слоя (в гетерофазной прослойке толщиной $D>2 d s$ ) представить как гомогенную смесь двух компонент: структурированного высоковязкого $\left(\mu_{s}\right)$ „ворса“ с его долей $(q)$ и менее вязкой „объемной“ $\left(\eta_{\mathrm{vol}}\right)$ изотропной жидкости - с относительным ее содержанием в слое $(1-q)$. Его вязкость $\mu$ определяют вязкости компонент и их доли в слое. С возрастанием скорости сдвигового течения $\gamma\left(\mathrm{s}^{-1}\right)$ уменьшаются и модельные параметры $\left(q, \mu_{s}\right)$, и различие между $\mu_{s}$ и $\eta_{\mathrm{vol}}$. Величина вязкости слоя $\mu$ может быть рассчитана как вязкость смеси [20,21], и в самом простейшем приближении аддитивности текучестей компонент [22] определяется этими параметрами так:

$$
\mu=\mu_{s} \eta_{\mathrm{vol}} /\left[\mu_{s}-q\left(\mu_{s}-\eta_{\mathrm{vol}}\right)\right] .
$$

В прослойке $\left(d_{s}<D<2 d_{s}\right)$ ЭЖК слои каждой из подложек „перекрываются“, а в прослойке $D \leq d_{s}$ уже находится лишь „перекрытый“ ЭЖК слой вязкостью $\mu^{*}$, которая по аналогии с (1) имеет вид

$$
\mu^{*}=\mu_{s}^{*} \eta_{\mathrm{vol}} /\left[\mu_{s}^{*}-q^{*}\left(\mu_{s}^{*}-\eta_{\mathrm{vol}}\right)\right],
$$

где $\mu_{s}^{*}$ и $q^{*}$ - параметры, аналогичные использованным в (1), но для „Перекрытого“ ЭЖК слоя. Вязкость его

\footnotetext{
${ }^{2}$ Интересно, что еще ранее [18] граничный слой смазки был уподоблен ,бархатному ворсу“.

3 Таким образом, параметр $q$ - аналог параметра ориентационного порядка $S$ в ЖК [19] и ЭЖК $[2,3]$, который также изменяется от $S=1$ - максимально упорядоченный ЖК, до $S=0-$ изотропная жидкость.
} 


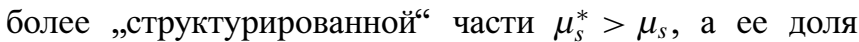
$q^{*}>q$ (за счет частичного „вытеснения“ изотропной жидкости в области перекрытия), поэтому и $\mu^{*}>\mu$.

\section{Реологическая модель гетерофазной прослойки с ЭЖК слоями}

Для установления параметров ЭЖК слоя при обработке результатов вискозиметрии прослоек здесь используется их „реологическая“ модель $[14,15]$, в которой (как и в моделях „оптических“ $[3,12])$ слой однороден и характеризуется неизменным по всей толщине $d_{s}$ коэффициентом вязкости $\mu$ (в „оптических“ - коэффициентами поглощения и двулучепреломления света). Сдвиговое течение, „срезая“ $[15,16]$ ЭЖК слой, изменяет его толщину: $d_{s}(\gamma)$.

На рис. 1 показана схема такой прослойки толщиной $D \geq 2 d_{s}$ при отсутствии течения в ней $(a)$ и при течении $b$.

Между ЭЖК слоями I и III толщиной $d_{0 s}$ и вязкостью $\mu_{0}$ (при течении соответственно $d_{s}$ и $\mu$ ) находится

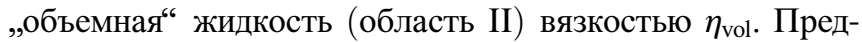
ставленный на рис. $1, b$ характер профиля скорости при
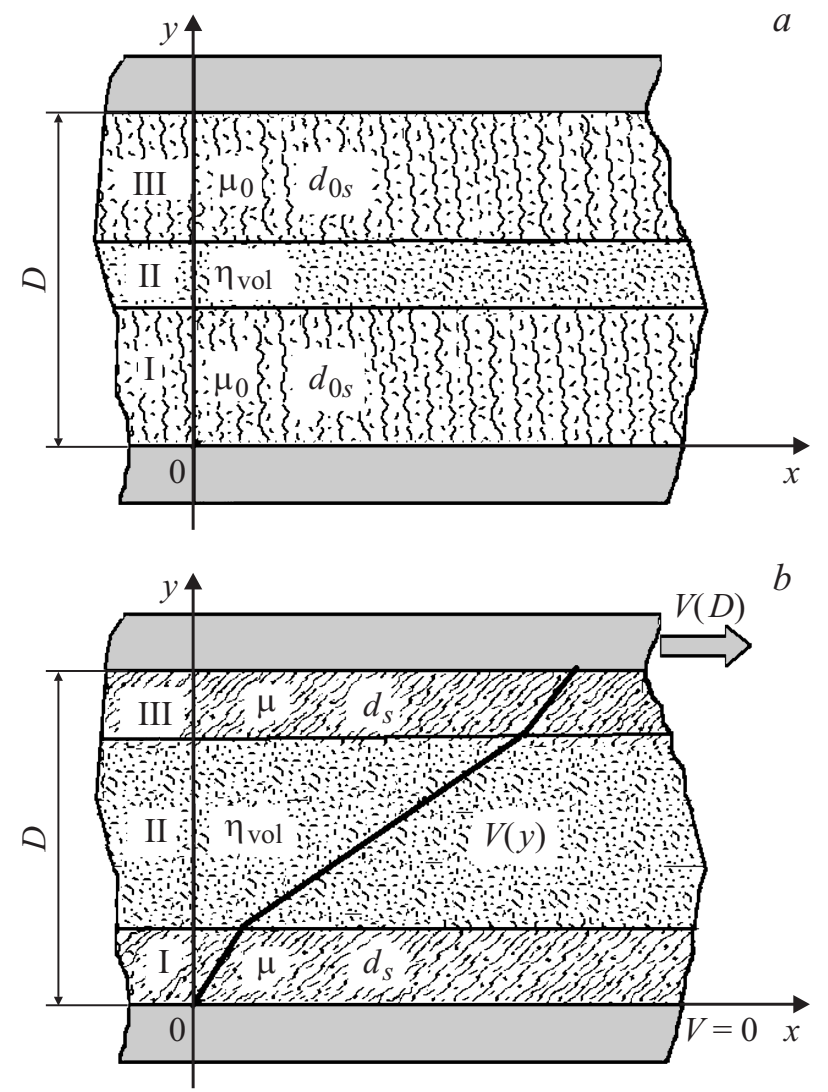

Рис. 1. Модель гетерофазной прослойки $D \geq 2 d_{s}$ в статике $(a)$ и с плоскопараллельным течением жидкости в ней $(b)$. В областях I и III - однородные ЭЖК слои, область II между ними - „объемная“ жидкость. $V(y)$ - профиль скорости течения.

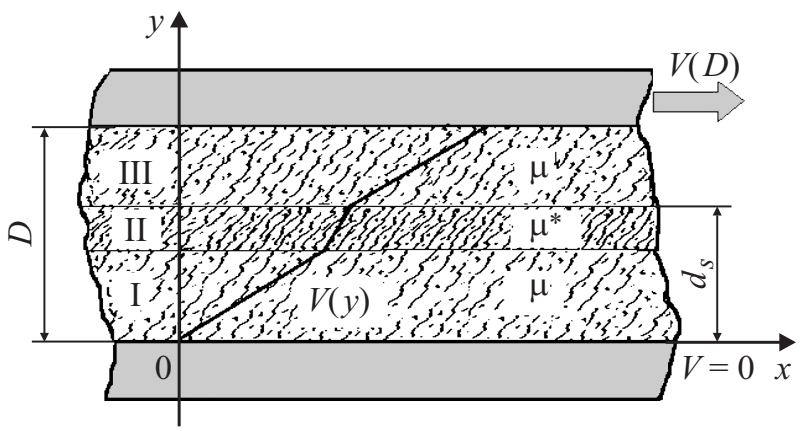

Рис. 2. Плоскопараллельное течение в гетерофазной прослойке $d_{s} \leq D \leq 2 d_{s}$. I и III - пристеночные однородные ЭЖК слои толщиной $D-d_{s}$ и вязкостью $\mu$, а II между ними „перекрытые“ ЭЖК слои вязкостью $\mu^{*} . V(y)-$ профиль скорости течения.

сдвиговом течении следует из решения [14] краевой задачи (уравнение Навье-Стокса с учетом сцепления текущей жидкости со стенками, условия сопряжения скоростей и вязких напряжений на границах областей). Там же [14] для такой реомодели гетерофазной прослойки получено простое соотношение, связывающее $d_{s}$ и $\mu$ с $D$ и $\eta_{\mathrm{eff}}$

$\eta_{\mathrm{eff}}(\gamma, D)=\eta_{\mathrm{vol}} /\left\{1-\left(1-\eta_{\mathrm{vol}} / \mu\right) 2 d_{s}(\gamma) / D\right\}\left(D \geq 2 d_{s}\right)$.

Очевидное возрастание $\eta_{\text {eff }}$ с уменьшением толщины прослойки $D$ (до $D=2 d_{s}$ ) связано с уменьшением в ней при этом в области II (рис. 1) менее вязкой изотропной жидкости. При утончении прослойки (начиная с $D=2 d_{s}$ и до $D=d_{s}$ ) в центральной ее области вместо изотропной жидкости появляется (рис. 2) область II „перекрытых“ ЭЖК слоев толщиной $\left(2 d_{s}-D\right)$ и вязкостью $\mu^{*}(2)$.

В этом случае решение аналогичной краевой задачи, но уже с другими вязкостями (рис. 2), имеет вид

$$
\begin{gathered}
\eta_{\mathrm{eff}}(\gamma, D)=1 /\left\{2 / \mu-1 / \mu^{*}-\left(1 / \mu-1 / \mu^{*}\right) 2 d_{s}(\gamma) D\right\} \\
\left(d_{s}<D<2 d_{s}\right)
\end{gathered}
$$

\section{Зависимость „предельной“ вязкости гетерофазной прослойки от ее толщины}

При отсутствии течения $(\gamma \rightarrow 0)$ слой характеризуется (рис. 1,a) „равновесной“ толщиной слоя $d_{0 s}=d_{s}(\gamma \rightarrow 0)$, неизменной (при постоянной $T$ ) в области толщин прослоек $D \geq 2 d_{0 s}$. Поэтому „предельная“ эффективная вязкость $\eta_{\mathrm{eff}}^{0}=\eta_{\mathrm{eff}}(\gamma \rightarrow 0)$ таких прослоек в изотермическом течении уже зависит лишь от их толщины $D$ :

$$
\eta_{\mathrm{eff}}^{0}(0, D)=\eta_{\mathrm{vol}} /\left\{1-\left(1-\eta_{\mathrm{vol}} / \mu_{0}\right) 2 d_{0 s} / D\right\} \quad\left(D \geq 2 d_{s}\right) .
$$

Из (5) следует, что задачу определения искомых модельных параметров $d_{0 s}, \mu_{0}$ (и $\left.\mu_{0}^{*}\right)$ можно решить, 
экспериментально измеряя „предельную“ вязкость $\eta_{\mathrm{eff}}^{0}$ прослойки известной толщины $D$. В предыдущих работах по реологии гетерофазных прослоек [12,14-16] определение $\eta_{\mathrm{eff}}^{0}(0, D)$ проводилось экстраполяцией массива экспериментальных данных $\eta_{\mathrm{eff}}(\gamma, D)$ к $\gamma=0$. Однако из (5) видно, что обратная безразмерная вязкость прослойки $\eta_{\mathrm{vol}} / \eta_{\mathrm{eff}}^{0}$ зависит от $1 / D$ линейно. Это позволяет находить искомые параметры слоя из вида экспериментального графика функции $\eta_{\mathrm{vol}} / \eta_{\mathrm{eff}}^{0}=F(1 / D)$ :

$$
\eta_{\mathrm{vol}} / \eta_{\mathrm{eff}}^{0}(0, D)=1-\left(1-\eta_{\mathrm{vol}} / \mu_{0}\right) 2 d_{0 s} / D \quad\left(D \geq 2 d_{s}\right)
$$

В самом деле, графически (6) отображает отрезок прямой, соединяющий (для всех $\Delta T$ ) точки $(0 ; 1)$ и $\left(1 / 2 d_{0 s} ; \eta_{\mathrm{vol}} / \mu_{0}\right)$. Начиная с последней, уже в области $2 d_{0 s} \geq D \geq d_{0 s} \quad$ (без „объемной“ $\eta_{\mathrm{vol}} / \eta_{\mathrm{eff}}^{0}=F(1 / D)$ в соответствии с (4) имеет вид

$$
\begin{aligned}
& \eta_{\mathrm{vol}} / \eta_{\mathrm{eff}}^{0}(0, D)=2 \eta_{\mathrm{vol}} / \mu_{0}-\eta_{\mathrm{vol}} / \mu_{0}^{*} \\
& \quad-\left(\eta_{\mathrm{vol}} / \mu_{0}-\eta_{\mathrm{vol}} / \mu_{0}^{*}\right) 2 d_{0 s} / D \quad\left(2 d_{s} \geq D \geq d_{s}\right) .
\end{aligned}
$$

Это уравнение, отражая изменение безразмерной вязкости прослойки уже за счет изменения области II (рис. 2) „перекрытых“ ЭЖК слоев, графически в тех же координатах также представляет собой отрезок прямой.

Наконец, начиная с точки $\left(1 / d_{0 s} ; \eta_{\text {vol }} / \mu_{0}^{*}\right)$ для зависимости от $1 / D$ безразмерной $\left(\eta_{\mathrm{vol}} / \mu_{0}^{*}\right)$ вязкости прослойки с ,перекрытым“ ЭЖК слоем при однородности последнего, можно также ожидать линейности. Следовательно, определение особенностей (изломов) ломаной $\eta_{\mathrm{vol}}(T) / \eta_{\mathrm{eff}}^{0}(0, D, T)=F(1 / D)$ решает задачу. Очевидно, что необходимые значения $\eta_{\mathrm{vol}} / \eta_{\mathrm{eff}}^{0}=F(1 / D)$ рациональнее получать экстраполяцией к $\gamma=0$ тренда $\eta_{\mathrm{vol}} / \eta_{\mathrm{eff}}(\gamma, D)=F(\gamma)$.

Таким образом, измерения величины $\eta_{\mathrm{eff}}(\gamma)$ эффективной вязкости прослойки (при различных ее толщинах и температурах) и описанная методика анализа результатов позволяют провести оценку модельных параметров слоя ЭЖК и установить влияние на них температуры и интенсивности сдвигового течения.

\section{Результаты измерений и их обсуждение}

Вискозиметром $[12,15]$ в зазорах $D(\sim 1-10 \mu \mathrm{m})$ его ротационной пары - аналоге триады трения при скоростях сдвиговых деформаций $\left(\gamma=100-15000 \mathrm{~s}^{-1}\right)$ в изотермических опытах при нескольких температурах (начиная с малых $\Delta T=T-T_{\text {cr }}=1-2 \mathrm{~K}$ и в интервале $\Delta T \sim 30 \mathrm{~K})$ измерялась эффективная вязкость $\eta_{\mathrm{eff}}(\gamma, D, T) \quad$ прослоек $\quad \boldsymbol{H}$-гексадекана ${ }^{4} \quad \mathrm{C}_{16} \mathrm{H}_{34}$ $\left(T_{\mathrm{cr}}=291.2 \mathrm{~K}\right)$.

В измерениях для ряда толщин (зазоров пары) прослоек $D$ были получены массивы $\eta_{\mathrm{eff}}=f(D, \gamma)$. Далее в соответствии с (6) и (7) устанавливалась зависимость

\footnotetext{
4 Дополнительно очищенный препарат производства Новочеркасского завода синтетических продуктов квалификации „ХЧ“.
}

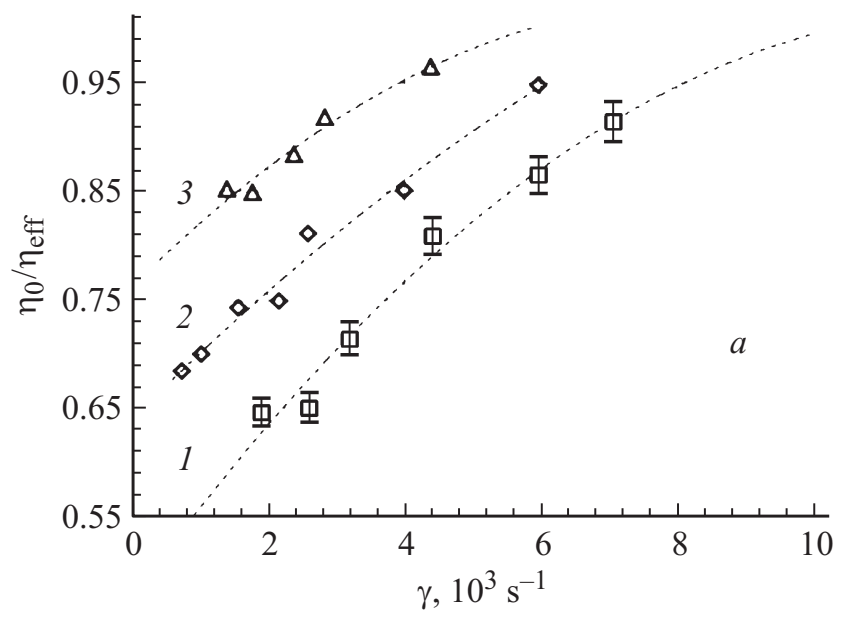

Рис. 3. Реокривые прослоек н-гексадекана (при $\Delta T=1.5 \mathrm{~K}$ ) между стальными подложками толщиной $D(\mu \mathrm{m}) \approx 1-6$, $2-4,3-1.5$. Использован график [16, рис. $1, a]$, перестроенный в координатах $\eta_{0} / \eta_{\mathrm{eff}}\left(\gamma_{i}, D, T\right)=F\left(\gamma_{i}\right)$.

от $\gamma$ „относительной“ вязкости: $\eta_{\text {vol }}(T) / \eta_{\mathrm{eff}}(T, \gamma)=F(\gamma)$. В результате ее экстраполяции к $\gamma \rightarrow 0$ определялись необходимые (для построения графиков) значения $\eta_{\mathrm{vol}}(T) / \eta_{\mathrm{eff}}^{0}(0, D, T)$. Заметим, что в функции $F(\gamma)$ одновременно учитываются температурные зависимости $\eta_{\mathrm{vol}}(T)$ и $\eta_{\mathrm{eff}}(T)$, а благодаря иному виду тренда $F(\gamma)$ его экстраполяция позволяет устанавливать эти значения и более надежно. Так, одна из проведенных в [16] серий измерений вязкости прослоек $H$-гексадекана $(D \approx 6$; 4, и $1.5 \mu \mathrm{m}$ при $\left.\Delta T=T-T_{\mathrm{cr}}=1.5 \mathrm{~K}\right)$, которая была представлена $[16$, рис. $1, a]$ в виде $\eta_{\mathrm{eff}}(\gamma, D, T)=F(\gamma)$, здесь пересчитана (рис. 3) в виде зависимостей $\eta_{\mathrm{vol}} / \eta_{\mathrm{eff}}(\gamma, D, T)=F(\gamma)$.

При анализе результатов опытов использовались приведенные выше представления о слое ЭЖК и ожидаемые (из модели гетерофазной прослойки с ним) аналитические зависимости (6) и (7), Предельной“ эффективной вязкости прослойки от ее толщины.

Предельные значения $\eta_{\text {vol }} / \eta_{\mathrm{eff}}^{0}(0, D, T)$, полученные экстраполяцией к $\gamma \rightarrow 0$ реокривых $\eta_{\mathrm{vol}} / \eta_{\mathrm{eff}}(\gamma, D, T)=$ $=F(\gamma)$ всех серий измерений $[16]$, а также $[14,15]$ и других измерений ${ }^{5}$ вязкости прослоек $H$-гексадекана (при близких температурах), представлены на рис. 4 графиком $\eta_{\mathrm{vol}} / \eta_{\mathrm{eff}}^{0}=F(1 / D)$. На нем для четырех температур $\left(T-T_{\text {cr }}=1.5 ; 6.5 ; 12\right.$ и $\left.18 \mathrm{~K}\right)$ штриховыми линиями показаны тренды отрезков прямых, построенных с учетом того, что абсцисса первого излома графика $\left(1 / D=1 / 2 d_{0 s}\right)$ вдвое меньше второго $\left(1 / D=1 / d_{0 s}\right)$. Видно, что после этой точки $\left(1 / d_{0 s} ; \eta_{\mathrm{vol}}^{0} / \mu_{0}^{*}\right)$ при $\Delta T=1.5 \mathrm{~K}$ с уменьшением $D$ безразмерная $\left(\eta_{\mathrm{vol}} / \eta_{\mathrm{eff}}^{0}\right)$ вязкость прослойки практически не изменяется. ${ }^{6}$

\footnotetext{
5 Такие измерения выполняли также Н.А. Поляковская, А.А. Шатагина, Е.А. Шатагина и И.А. Шатагин.

${ }^{6}$ При повышенных $\Delta T$ ввиду особой малости $D$ установить это надежно нашим вискозиметром затруднительно.
} 


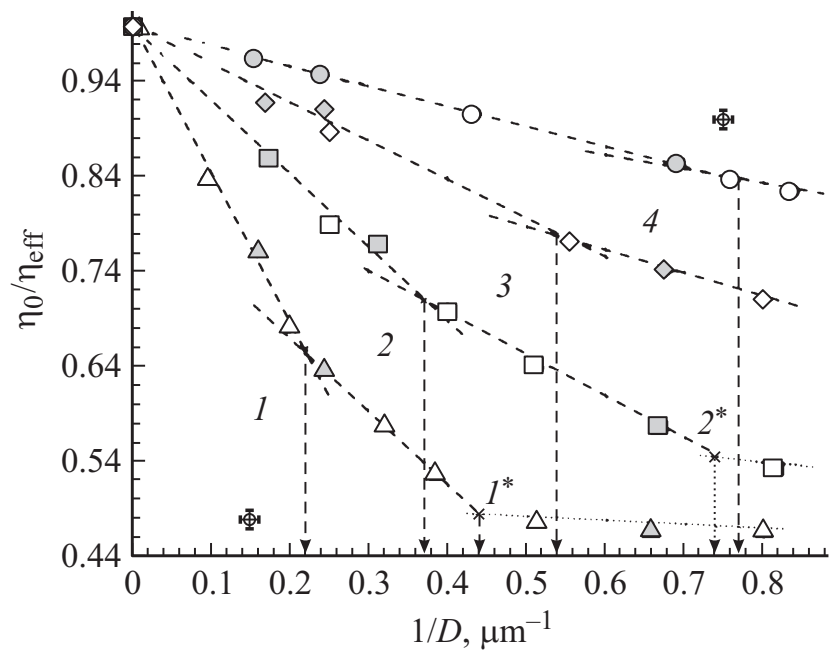

Рис. 4. Экспериментальные зависимости „предельной“ $(\gamma \rightarrow 0)$ относительной вязкости $\eta_{\mathrm{vol}} / \eta_{\mathrm{eff}}^{0}$ прослоек $H^{-}$ гексадекана от их обратной толщины $1 / D$. 1-4 - значения $\eta_{\mathrm{vol}} / \eta_{\mathrm{eff}}^{0}=\eta_{\mathrm{vol}} / \mu_{0}$ при $D=2 d_{0 s} .1^{*}, 2^{*}-$ значения $\eta_{\mathrm{vol}} / \eta_{\mathrm{eff}}^{0}=\eta_{\mathrm{vol}} / \mu_{0}^{*}$ при $D=d_{0 s}$. Измерения проведены при $T-T_{\text {cr }}(\mathrm{K}): \Delta-1.5, \square-6.5, \diamond-12, \circ-18$ соответственно. Залитые маркеры - результаты опытов [16] с н-гексадеканом.

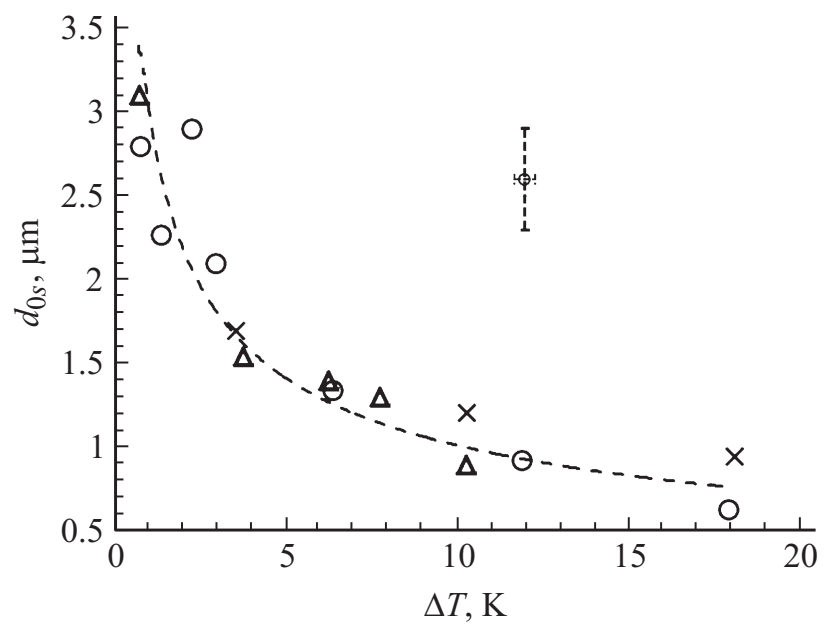

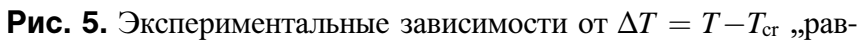
новесной“ толщины $d_{0 s}$ ЭЖК слоя $н$-гексадекана на стали из измерений: о - вязкости ротационным вискозиметром, $\Delta-$ оптической анизотропии (ДЛП) и $\times-$ дихроизма (методом „гость-хозяин“) его прослоек.

Установленные в этих и других измерениях вязкости прослоек $н$-гексадекана значения „равновесной“ толщины $d_{0 s}(\mu \mathrm{m}, \delta \approx 10 \%)$ его ЭЖК слоя на стали показаны на рис. 5. Представленная на нем зависимость $d_{0 s}$ от температуры (в интервале $\Delta T \approx 1-20 \mathrm{~K}$ ) построена с учетом и измерений $d_{0 s}(\Delta T)$ в гетерофазных прослойках $\boldsymbol{H}$-гексадекана оптической анизотропии (в „щелевом световоде“ ${ }^{\text {“ }}$, и дихроизма (методом „гость-хозяин“) [3].

Как видно из рис. 5, толщина ЭЖК слоя $н$-гексадекана вблизи температуры кристаллизации жидкости состав- ляет $d_{0 s} \approx 3-4 \mu \mathrm{m}$, уменьшается с повышением температуры - удалением от $T_{\mathrm{cr}}$, а при $\Delta T>25 \mathrm{~K}$ слоя практически уже нет. Видно также, что результаты измерений этого параметра ЭЖК слоя $H$-гексадекана на стали другими методами в пределах погрешности совпадают.

Отметим, что и для смазочных масел „равновесная“ толщина ЭЖК слоев на стали того же порядка и убывает с температурой, но медленней $[12,14,15]$. Так, для масла 15W40: $d_{0 s} \approx 3 \mu \mathrm{m}(T=291 \mathrm{~K})$ и $d_{0 s} \approx 2 \mu \mathrm{m}$ $(T=304 \mathrm{~K})$ [12]. На диэлектрической же подложке (кварц) их ЭЖК слои значительно $\left(d_{0 s} \approx 0.2 \mu \mathrm{m}\right)$ тоньше $[2,3]$.

Зависимости „предельных“ $(\gamma \rightarrow 0)$ коэффициентов вязкости $\left(\mathrm{mPa}^{*} \mathrm{~s}\right)$ ЭЖК слоя $\boldsymbol{H}$-гексадекана - $\mu_{0}$ $(\delta \approx 7 \%)$, „перекрытого“ $-\mu_{0}^{*}(\delta \approx 10 \%)$ и в „объеме“ $-\eta_{\text {vol }}$, от температуры $\left(\Delta T=T-T_{\mathrm{cr}}, \mathrm{K}, \delta \approx 1 \%\right)$

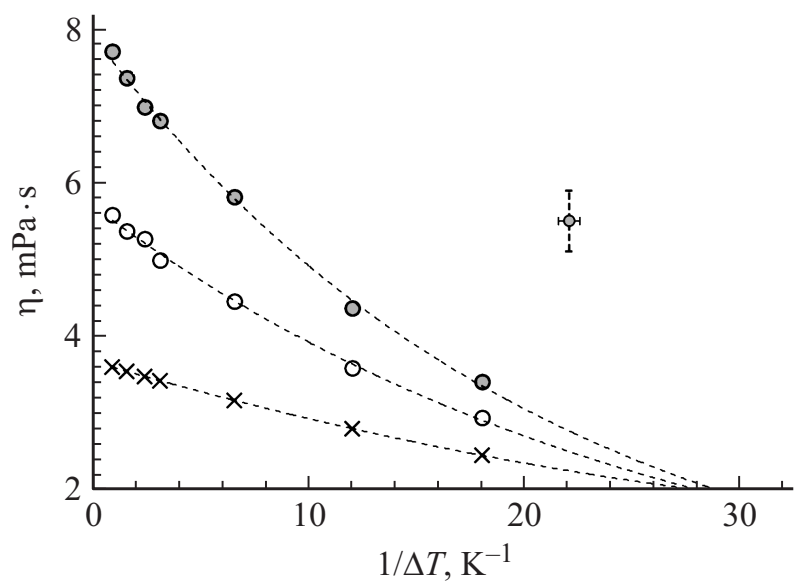

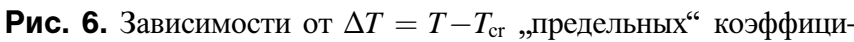
ентов вязкости ЭЖК слоев $H$-гексадекана на стали. о - $\mu_{0}$ (при $\left.D=2_{d s}\right), \bullet-\mu_{0}^{*}$, ,Перекрытого“ слоя (при $\left.D=d_{s}\right), \times-\eta_{\text {vol }}$ вязкость $H$-гексадекана в „объеме“.

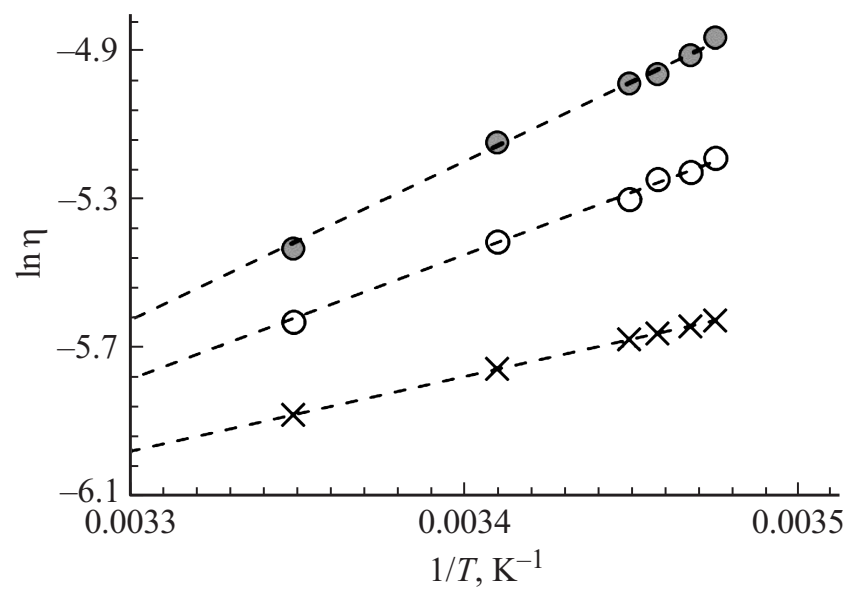

Рис. 7. Зависимость логарифма коэффициентов вязкости $\ln (\eta)=F(1 / T) н$-гексадекана в „объеме“ $\left(\eta=\eta_{\mathrm{vol}}\right)-\times$, и на стальной подложке его ЭЖК слоя $\left(\eta=\mu_{0}^{*}\right)-$ о; ЭЖК слоя „перекрытого“ $\left(\eta=\mu_{0}^{*}\right)-\bullet$. 
показаны на рис. 6. Последняя зависимость $\eta_{\mathrm{vol}}(\Delta T)$, полученная в наших опытах, близка $(\sim 3 \%)$ к литературным данным [23] в этом же интервале температур. Как видно из рис. 6 , вязкость слоя $\mu_{0}$ выше объемной $\eta_{\text {vol }}, \mu_{0}^{*}$ - еще выше, но с ростом температуры и они и их различие уменышается, а при $\Delta T \approx 30 \mathrm{~K}$ все три коэффициента вязкости уже одинаковы.

Установленные температурные зависимости вязкости $\mu_{0}(T)$ и $\mu_{0}^{*}(T)$ позволили также оценить (как и [16] аппроксимацией их экспонентами [24]) и величины энергий активации вязкого течения $E$ ЭЖК слоя и слоя „перекрытого“ $-E^{*}$. Графики таких аппроксимаций для них и для н-гексадекана в „объеме“ в полулогарифмических координатах $\ln (\eta)=F(1 / T)$ представлены на рис. 7. Линии всех трех зависимостей сходятся при $T \approx 325 \mathrm{~K}(\Delta T \approx 34 \mathrm{~K})$. Заметим, что для гетерофазных прослоек $н$-гексадекана те же зависимости существенно непрямолинейны [16].

Рассчитанные величины $E(\delta E \sim 10 \%)$ составляют $E=3360 \mathrm{~K}$ и $E^{*}=4280 \mathrm{~K}$. То, что энергия активации вязкого течения $H$-гексадекана в „объеме“ жидкости $\left(E_{\mathrm{vol}}=2018 \mathrm{~K}\right)$ меньше (в 1.7) энергии $E$ ЭЖК слоя, обусловлено структурированностью последнего. С нею связано и то, что величина $E^{*}$ „перекрытого“ и более структурированного слоя еще (в 2.1 раза) выше.

Заметим, что установленные зависимости $\mu_{0}(T)$ и $\mu_{0}^{*}(T)$ в принципе позволяют установить и соответствующие зависимости модельных параметров ЭЖК слоя $\mu_{s}(T)$ и $\mu_{s}^{*}(T)$. Для этого необходимо измерить (например, методом дихроизма $[2,3])$ при нескольких температурах величину $S$ ориентационного порядка в ЭЖК слое (и слое ,Перекрытом“) и, используя отмеченную аналогию $S$ и $q$, определить $\mu_{s}$ и $\mu_{s}^{*}$ из соотношений (1) и (2).

\section{Заключение}

В предложенной структурно-реологической модели ЭЖК слой состоит из высоко структурированной фазы и изотропной жидкости. Применение модели для анализа результатов измерений эффективной вязкости гетерофазных прослоек (при различных ее толщинах и температурах) позволяет установить влияние температуры на важные эксплуатационные параметры масляной прослойки в триаде трения: определяющую противоизносные свойства смазки, толщину ее ЭЖК слоя и особенности вязкости последнего. С использованием модели для одного из $н$-алканов (основ минеральных смазочных масел) - $\boldsymbol{H}$-гексадекана уточнен характер уменьшения с температурой равновесной толщины его ЭЖК слоя на стальной подложке. Вязкость его вследствие структурированности выше вязкости „объемной“ жидкости. В тонких прослойках ЭЖК слои от каждой из подложек образуют „перекрытый“ слой еще большей вязкости. Оба слоя отличаются и значительно более высокими энергиями активации вязкого течения.

\section{Список литературы}

[1] Дерягин Б.В., Чураев Н.В., Муллер В.М. Поверхностные силы. М.: Наука, 1985. 398 с.

[2] Derjaguin B.V., Altoiz B.A., Nikitenko I.I. // J. Colloid Interf. Sci. 1991. Vol. 145. N 2. P. 441-446.

[3] Алтоиз Б.А., Поповский Ю.М. Физика приповерхностных слоев жидкости. Одесса: Астропринт, 1996. 153 с.

[4] Lyakhova E. // J. Mol. Liquids. 2001. N 93. P. 203-206.

[5] Mikhailenko V.I., Popovskii A.Yu., Kuznetsova A.A. // J. Appl. Spectr. 2006. Vol. 73. N 4. P. 443-446.

[6] Kuznetsova A.A., Mikhailenko V.I., Popovskii A.Y. // J. Appl. Spectr. 2004. Vol. 71. N 5. P. 648-653.

[7] Алтоиз Б.А., Бондарев В.Н., Шатагина Е.А., Кириян С.В. // ЖТФ. 2014. Т 84. Вып. 7. С. 58-61.

[8] Петров А.А. Углеводороды нефти. М.: Наука, 1974. 264 с.

[9] Сюняев 3.И. Нефтяные дисперсные системы. М.: Химия, 1990. $226 \mathrm{c}$.

[10] Фукс Г.И. Вязкость и пластичность нефтепродуктов. Москва; Ижевск: Институт компьютерных исследований, 2003. $328 \mathrm{c}$.

[11] Мур Д. Основы и применения трибоники. М.: Мир, 1978. $488 \mathrm{c}$.

[12] Кириян С.В., Алтоиз Б.А. // Трение и износ. 2012. Т.33. Вып. 4. С. 367-372.

[13] Сайдов Г.В., Юдович М.Е. // Опт. и спектр. 1974. Т. 36. C. $1216-1217$.

[14] Алтоиз Б.А., Кириян С.В., Шатагина Е.А. // ЖТФ. 2010. T. 80. Вып. 10. С. 37-40.

[15] Алтоиз Б.А., Кириян С.В. // ИФЖ. 2010. Т. 83. Вып. 3. C. 608-613.

[16] Шатагина А.А., Шатагина Е.А., Шатагин И.А., Бутенко А.Ф. // Физика аэродисперсных систем. 2015. № 52. C. $22-27$.

[17] Межиковский С.М. Олигомерное состояние вещества. М.: Наука, 2005. 252 с.

[18] Finch G.I. // Structure and Formation of Thin Films. 1937. Vol. 56. N 28. P. 632-639.

[19] Жен П. Физика жидких кристаллов. М.: Мир, 1977. 396 с.

[20] Robert C. Reid, John M. Prausnitz, Thomas K. Sherwood. The properties of gases and liquids. NY: McGraw-Hill Book Company, 1977. 704 p.

[21] Boris Zhmud. Viscosity blending equations // The Europ. Lubricants industry magazine. 2014. N 93. P. 1-4.

[22] Рабинович Г.Г., Рябых П.М., Хохряков П.А. и др. / Под ред. Е.Н. Судакова. Расчеты основных процессов и аппаратов нефтепереработки: Справочник / 3-е изд., перераб. и доп. М.: Химия, 1979. 568 с.

[23] Физико-химические свойства индивидуальных углеводородов / Под ред. В.М. Татевского. М., 1960. 412 c.

[24] Виноградов Г.А., Малкин А.Я. Реология полимеров. М.: Химия, 1977. $439 \mathrm{c.}$ 